\title{
Cinchona Alkaloid-Catalyzed Asymmetric Conjugate Additions: The Bifunctional Brønsted Acid-Hydrogen Bonding Model
}

\author{
Matthew N. Grayson ${ }^{\dagger, \ddagger}$ and K. N. Houk ${ }^{*}, \dagger$ \\ Department of Chemistry and Biochemistry, University of California, Los Angeles, California 90095-1569, United States. \\ tCentre for Molecular Science Informatics, Department of Chemistry, University of Cambridge, Lensfield Road, Cambridge \\ CB2 1EW, United Kingdom.
}

\section{Supporting Information}

\begin{abstract}
Wynberg's report from 1977 that natural cinchona alkaloids catalyze the asymmetric conjugate addition of aromatic thiols to cycloalkenones is a landmark discovery in hydrogen bonding organocatalysis. Wynberg proposed that this reaction proceeded via the formation of a thiolate-alkylammonium tight ion pair and activation of the enone electrophile by a hydrogen bond from the catalyst's hydroxyl group. This reaction model provided the mechanistic basis for understanding Wynberg's reaction and many other asymmetric transformations since. Our quantum mechanical calculations reveal a different model should be used to explain the results: the alkylammonium ion activates the enone by Brønsted acid catalysis and the catalyst's hydroxyl group orients the thiolate nucleophile. The new model rationalizes the stereoselective outcome of Wynberg's reaction and provides a new, general model for asymmetric cinchona organocatalysis.
\end{abstract}

Wynberg reported in 1977 that cinchona alkaloids catalyze the asymmetric conjugate additions of aromatic thiols to cycloalkenones (Figure 1). ${ }^{1,2}$ This discovery has been regarded as a landmark in hydrogen bonding asymmetric organocatalysis. Since this pioneering work, cinchona alkaloids and their derivatives have been found to catalyze a multitude of asymmetric carboncarbon and carbon-heteroatom bond forming reactions ${ }^{3-5}$ that afford versatile building blocks for the synthesis of biologically active natural products and pharmaceutical compounds. ${ }^{6}$

Wynberg proposed that this reaction proceeds via the formation of a thiolate-quinuclidinium tight ion pair and that the enone electrophile is activated by a hydrogen bond from the catalyst's hydroxyl group, leading to the formation of a hydrogen bondstabilized oxyanion after carbon-sulfur bond formation (Mode A, Figure 1). This reaction model provided the basis for understanding Wynberg's reaction and many others since ${ }^{7-9}$ and has remained unchallenged for 35 years. This transition state (TS) model was based on the results of NOESY NMR experiments and molecular docking studies. ${ }^{10}$ Experimental and computational techniques that investigate the structure of intermediates present in reaction mixtures are commonly used to gain insight into reaction mechanisms. However, these results can be misleading, because the preferred TS complex is not necessarily derived from the lowest energy complex present in solution. ${ }^{11-14}$

Both theoretical and experimental mechanistic studies of reactions catalyzed by cinchona alkaloid derivatives have been reported. ${ }^{15-27}$ Cucinotta et al. studied the conjugate addition of 1,3dicarbonyl compounds to maleimides catalyzed by natural cinchona alkaloids with density functional theory (DFT), but only one possible bifunctional activation mode was considered, the pathway originally proposed by Wynberg. ${ }^{7}$ Hintermann et al. studied a natural cinchona alkaloid-catalyzed oxa-Michael cyclization using experimental techniques which provided some evidence for concerted $\mathrm{O}-\mathrm{C}$ and $\mathrm{C}-\mathrm{H}$ bond formation but other mechanistic possibilities could not be fully excluded. ${ }^{8}$ This lack of clear mechanistic understanding prevents the rational design of new reactions and limits catalyst development in what is an immensely important area of catalysis.

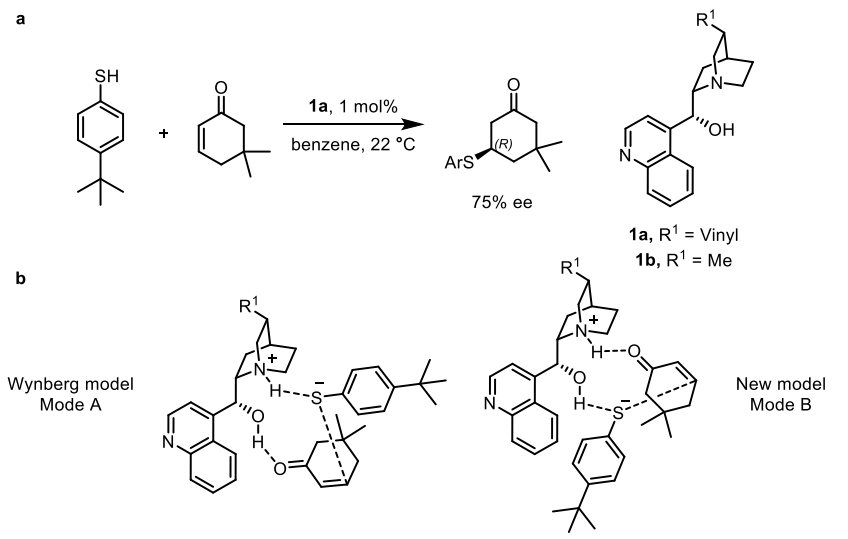

Figure 1. Wynberg's cinchonidine-catalyzed asymmetric conjugate addition of aromatic thiols to cycloalkenones. a, Experimental reaction conditions. $b$, Possible activation modes.

We have carried out DFT calculations that show that the preferred complex of reactants is the ion pair that was suggested by Wynberg. However, the lowest energy TS leading to the major product proceeds through a different pathway (Mode B, Figure 1). In this new reaction model, the enone electrophile is activated by Brønsted acid catalysis and the hydroxyl group orients the thiolate nucleophile.

The vinyl group on the quinuclidine ring was replaced by a methyl group to simplify our calculations (catalyst 1b, Figure 1). This truncation has been reported to have minimal effect on the reaction outcome in related transformations catalyzed by cinchona alkaloid derivatives. ${ }^{15,16}$ Previous computational studies have shown that deprotonations of acidic nucleophiles by tertiary amines are facile. ${ }^{24,25}$ In our calculations, the quinuclidine nitrogen is protonated. In the base-catalyzed addition of thiolates to enones, the rate determining step is carbon-sulfur bond formation. ${ }^{28}$ Sulfa-Michael additions can be reversible, but Wynberg showed that the formation of the product in the reaction of thio- 
phenol and 2-cyclohexen-1-one catalyzed by quinine in toluene at $25{ }^{\circ} \mathrm{C}$ is kinetically controlled. ${ }^{2}$

In order to explore Wynberg's proposal that the lowest energy complex in solution is the tight ion pair formed between the quinuclidinium ion and thiolate, complexes between the enone, thiolate and protonated catalyst were optimized, and 73 unique complexes were located at the M06-2X/def2TZVPP-IEFPCM(benzene)//M06-2X/6-

$31 \mathrm{G}(\mathrm{d})-$ IEFPCM(benzene) level of theory ${ }^{29,30}$ using Gaussian $09^{31}$ (see Supporting Information for full computational details). The lowest energy one, Complex 1 (Figure 2), includes the quinuclidinium ion-thiolate ion pair and a hydrogen bond from the catalyst's hydroxyl group to the enone oxygen, in accord with Wynberg's model. An interaction between a $\mathrm{CH}$ of the quinoline ring and the thiolate is also present (2.70 $\AA$, Complex 1, Figure 2). The lowest energy Mode B complex was disfavored by $5.5 \mathrm{kcal}$ $\mathrm{mol}^{-1}$ (Complex 2, Figure 2).

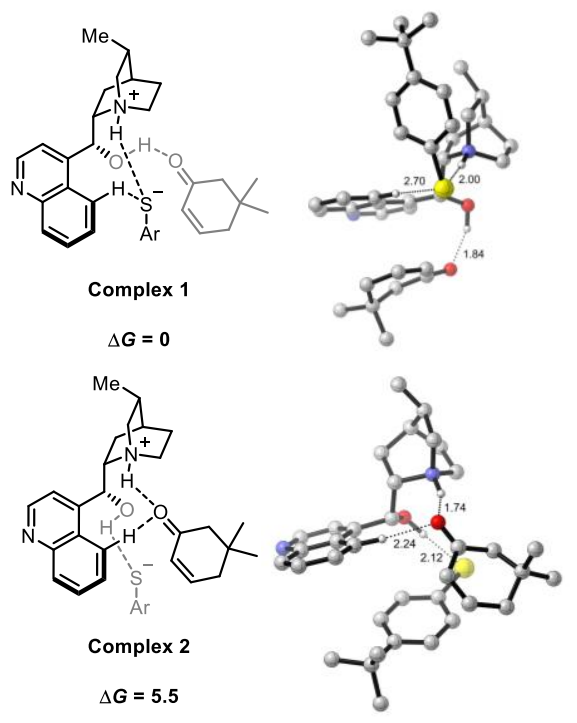

Figure 2. Pre-reaction complexes in Wynberg's cinchonidinecatalyzed asymmetric conjugate addition of aromatic thiols to cycloalkenones. M06-2X/def2-TZVPP-IEFPCM(benzene)//M062X/6-31G(d)-IEFPCM(benzene). Non-critical hydrogen atoms omitted for clarity. All energies in $\mathrm{kcal} \mathrm{mol}^{-1}$.

Figure 3 shows the lowest energy TSs leading to the major and minor products for the Wynberg ion pair-hydrogen bonding model (Mode A) and the Brønsted acid-hydrogen bonding model (Mode B). The anti-open conformation of the cinchonidine catalyst is present in all of these TSs. ${ }^{32,33}$ To verify that other catalyst conformations were disfavored, one Mode B TS was located for each of the other five possible catalyst conformations. They were calculated to be disfavored by $6.0 \mathrm{kcal} \mathrm{mol}^{-1}$ or more.

TS-B(major), which correspond to activation Mode B, is the lowest energy TS and leads to the major product observed experimentally. From the lowest energy complex, the free energy barrier to this TS is $12.8 \mathrm{kcal} \mathrm{mol}^{-1}$. The aromatic group of the thiolate prefers to be pointed away from the cyclohexanone. The lowest energy Mode A TS is $2.2 \mathrm{kcal} \mathrm{mol}^{-1}$ higher in energy (TSA(minor), Figure 3). The large geometry change between complex and TS is due to the developing alkoxide in the TS which is stabilized to a greater extent by the quinuclidinium ion in Mode B relative to the hydroxyl group in Mode A. The Mulliken atomic charge on the enone oxygen in TS-B(major) is -0.50 (calculated at the M06-2X/def2-TZVPP-IEFPCM(benzene) level of theory). $\mathrm{C}-\mathrm{S}$ bond formation and proton transfer from the quinuclidinium ion to the enone occur simultaneously. Simón and Paton have observed simultaneous carbon-carbon bond formation and proton transfer in the asymmetric phosphazene-catalyzed nitro- and phospho-aldol additions. ${ }^{34}$ An interaction between a $\mathrm{CH}$ of the quinoline ring and the oxygen of the enone also helps to stabilize the developing alkoxide in the TS $(2.27 \AA$, TS-B(major), Figure $3)$. This $\mathrm{CH}$... O interaction is worth approximately $1.6 \mathrm{kcal} \mathrm{mol}^{-1}$ (See Figure S2). The precursor reactant complex to TS-B(major) was located by IRC calculation and was calculated to be disfavored by $7.3 \mathrm{kcal} \mathrm{mol}^{-1}$ relative to Complex 1 . However, these complexes are in rapid equilibrium and their relative thermodynamic stabilities do not determine the preferred reaction pathway (Curtin-Hammett conditions).
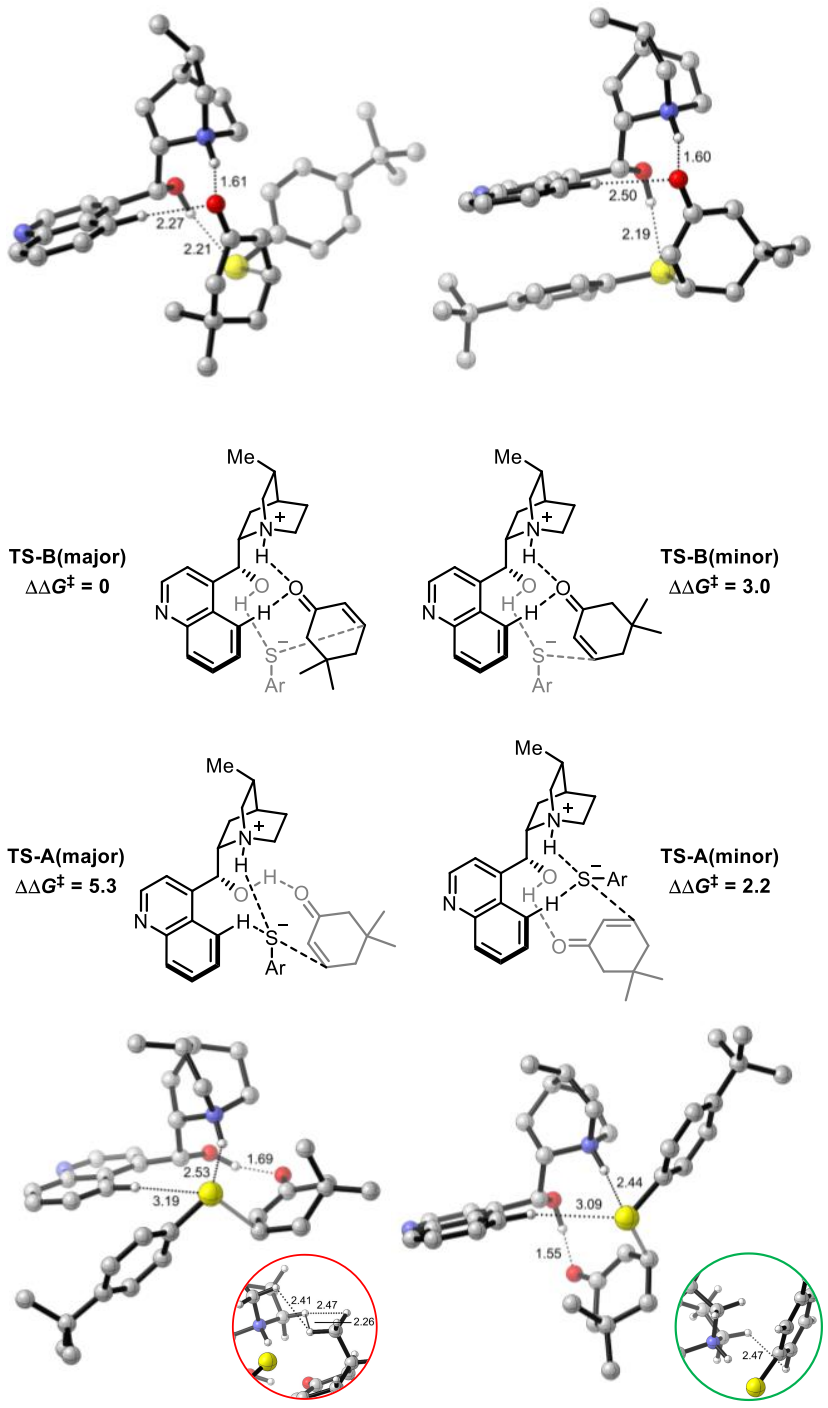

Figure 3. C-S bond forming TSs in Wynberg's cinchonidinecatalyzed asymmetric conjugate addition of aromatic thiols to cycloalkenones. M06-2X/def2-TZVPP-IEFPCM(benzene)//M062X/6-31G(d)-IEFPCM(benzene). Non-critical hydrogen atoms omitted for clarity. All energies in $\mathrm{kcal} \mathrm{mol}^{-1}$.

We also explored the origins of stereoselectivity. TS-B(minor) leads to the minor product via Mode B. This TS is destabilized relative to TS-B(major). While TS-B(major) proceeds via axial attack on the half-chair cyclohexanone, TS-B(minor) proceeds via equatorial attack leading to greater substrate distortion. Normally, axial attack is preferred, but for TS-B(minor) this would give an axial methyl group at $\mathrm{C}-5$ of the enone which clashes with 
the quinuclidine ring. TS-A(major) and TS-A(minor), which correspond to the ion pair-hydrogen bonding Mode A, are disfavored relative to TS-B(major), TS-A(major) is further destabilized relative to TS-A(minor) by a steric clash between the quinuclidine ring $\mathrm{CH}_{2}$ groups and an enone methyl group.

The concerted hydrogen bond network mechanism proposed by Hintermann et al. ${ }^{8}$ was also investigated. Eleven unique TSs were located, the lowest energy of which (TS-H(major)) is disfavored relative to TS-B(major) by $31.7 \mathrm{kcal} \mathrm{mol}^{-1}$ (Figure 4). Therefore, TSs of this nature were not investigated further.

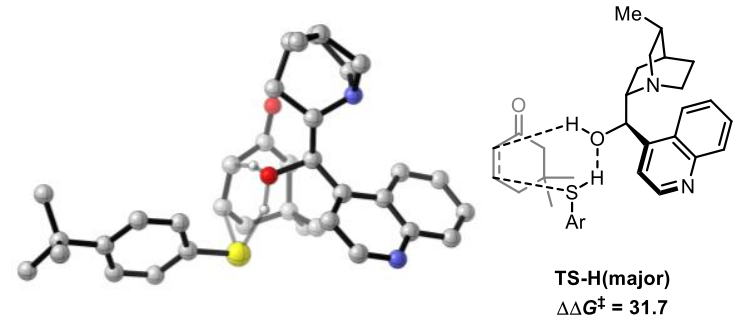

Figure 4. Alternative hydrogen bonded C-S bond forming TS in Wynberg's cinchonidine-catalyzed asymmetric conjugate addition of aromatic thiols to cycloalkenones. M06-2X/def2TZVPP-IEFPCM(benzene)//M06-2X/6-

$31 \mathrm{G}(\mathrm{d})-\mathrm{IEFPCM}$ (benzene). Free activation energy relative to TSB(major). Non-critical hydrogen atoms omitted for clarity. Energy in kcal mol-1.

In summary, Wynberg's original transition state model for the asymmetric conjugate additions of aromatic thiols to cycloalkenones was based on the results of NOESY NMR experiments and molecular docking studies. Indeed, the preferred pre-reaction complex is the ion pair proposed by Wynberg. However, the lowest energy TS leading to the major product observed experimentally involves proton transfer from the quinuclidinium ion to the enone and a hydroxyl group hydrogen bonding to the thiolate. The bifunctional Brønsted acid-hydrogen bonding model presented here will serve to promote further development of synthetic methodology involving bifunctional catalysts of this type.

\section{ASSOCIATED CONTENT}

\section{Supporting Information}

Complete list of authors in the Gaussian 09 reference, computational details and additional figures. Cartesian coordinates, energies, free energies and number of imaginary frequencies of all stationary points and values of imaginary frequencies of all transition structures. This material is available free of charge via the Internet at http://pubs.acs.org.

\section{AUTHOR INFORMATION}

\section{Corresponding Author}

houk@chem.ucla.edu

\section{Notes}

The authors declare no competing financial interests.

\section{ACKNOWLEDGMENT}

We are grateful to The English-Speaking Union (Lindemann Trust Fellowship to M.N.G.), Girton College, Cambridge (Research Fellowship to M.N.G.) and the NSF (CHE-1361104 to K.N.H.) for financial support. Computational resources were provided by the UCLA Institute for Digital Research and Education (IDRE) and the Extreme Science and Engineering Discovery En- vironment (XSEDE), which is supported by the NSF (OCI1053575). Part of this work was performed using the Darwin Supercomputer of the University of Cambridge High Performance Computing Service (http://www.hpc.cam.ac.uk/), provided by Dell Inc. using Strategic Research Infrastructure Funding from the Higher Education Funding Council for England and funding from the Science and Technology Facilities Council. We thank Yuhong Lam for helpful discussions regarding this work.

\section{REFERENCES}

(1) Helder, R.; Arends, R.; Bolt, W.; Hiemstra, H.; Wynberg, H. Tetrahedron Lett. 1977, 18, 2181-2182.

(2) Hiemstra, H.; Wynberg, H. J. Am. Chem. Soc. 1981, 103, 417-430.

(3) Doyle, A. G.; Jacobsen, E. N. Chem. Rev. 2007, 107, 5713-5743.

(4) Connon, S. J. Chem. Commun. 2008, 2499-2510.

(5) Melchiorre, P. Angew. Chem., Int. Ed. 2012, 51, 9748-9770.

(6) Jiang, L.; Chen, Y.-C. Catal. Sci. Technol. 2011, 1, 354.

(7) Cucinotta, C. S.; Kosa, M.; Melchiorre, P.; Cavalli, A.; Gervasio, F. L. Chem.-Eur. J. 2009, 15, 7913-7921.

(8) Hintermann, L.; Ackerstaff, J.; Boeck, F. Chem.-Eur. J. 2013, 19, 2311-2321.

(9) Li, H.; Wang, Y.; Tang, L.; Deng, L. J. Am. Chem. Soc. 2004, 126, 9906-9907.

(10) Dijkstra, G. D. H.; Kellogg, R. M.; Wynberg, H. Recl. Trav. Chim. Pays-Bas 1989, 108, 195-204.

(11) Halpern, J. Science 1982, 217, 401-407.

(12) Paton, R. S.; Goodman, J. M.; Pellegrinet, S. C. Org. Lett. 2009, 11, $37-40$.

(13) Grayson, M. N.; Goodman, J. M. J. Org. Chem. 2013, 78, 87968801.

(14) Grayson, M. N.; Goodman, J. M. J. Org. Chem. 2015, 80, 20562061.

(15) Lam, Y.-h.; Houk, K. N. J. Am. Chem. Soc. 2014, 136, 9556-9559.

(16) Lam, Y.-h.; Houk, K. N. J. Am. Chem. Soc. 2015, 137, 2116-2127.

(17) Dedeoglu, B.; Catak, S.; Yildirim, A.; Bolm, C.; Aviyente, V. ChemCatChem 2015, 7, 4173-4179.

(18) Li, H.; Liu, X.; Wu, F.; Tang, L.; Deng, L. Proc. Natl. Acad. Sci. U. S. A. 2010, 107, 20625-20629.

(19) Yang, H.; Wong, M. W. J. Am. Chem. Soc. 2013, 135, 5808-5818.

(20) Xue, X.-S.; Li, X.; Yu, A.; Yang, C.; Song, C.; Cheng, J.-P. J. Am. Chem. Soc. 2013, 135, 7462-7473.

(21) Jiang, H.; Zhang, G.; Liu, Y.; Guo, X.; Liu, Z.; Yan, H.; Liu, H.; Huang, X. Struct. Chem. 2015, 26, 951-959.

(22) Celebi-Olçüm, N.; Aviyente, V.; Houk, K. N. J. Org. Chem. 2009, 74, 6944-6952.

(23) Yang, W.; Tan, D.; Li, L.; Han, Z.; Yan, L.; Huang, K.-W.; Tan, C.H.; Jiang, Z. J. Org. Chem. 2012, 77, 6600-6607.

(24) Hamza, A.; Schubert, G.; Soós, T.; Papai, I. J. Am. Chem. Soc. 2006, $128,13151-13160$.

(25) Zhu, J.-L.; Zhang, Y.; Liu, C.; Zheng, A.-M.; Wang, W. J. Org. Chem. 2012, 77, 9813-9825.

(26) Hammar, P.; Marcelli, T.; Hiemstra, H.; Himo, F. Adv. Synth. Catal. 2007, 349, 2537-2548.

(27) Tan, B.; Lu, Y.; Zeng, X.; Chua, P. J.; Zhong, G. Org. Lett. 2010, 12, 2682-2685.

(28) Krenske, E. H.; Petter, R. C.; Zhu, Z.; Houk, K. N. J. Org. Chem. 2011, 76, 5074-5081.

(29) Simón, L.; Goodman, J. M. Org. Biomol. Chem. 2011, 9, 689-700.

(30) Overvoorde, L. M.; Grayson, M. N.; Luo, Y.; Goodman, J. M. J. Org. Chem. 2015, 80, 2634-2640.

(31) Frisch, M. J., et al. Gaussian 09; Gaussian, Inc., Wallingford, CT, 2013.

(32) Bürgi, T.; Baiker, A. J. Am. Chem. Soc. 1998, 120, 12920-12926.

(33) Olsen, R. A.; Borchardt, D.; Mink, L.; Agarwal, A.; Mueller, L. J.; Zaera, F. J. Am. Chem. Soc. 2006, 128, 15594-15595.

(34) Simón, L.; Paton, R. S. J. Org. Chem. 2015, 80, 2756-2766. 


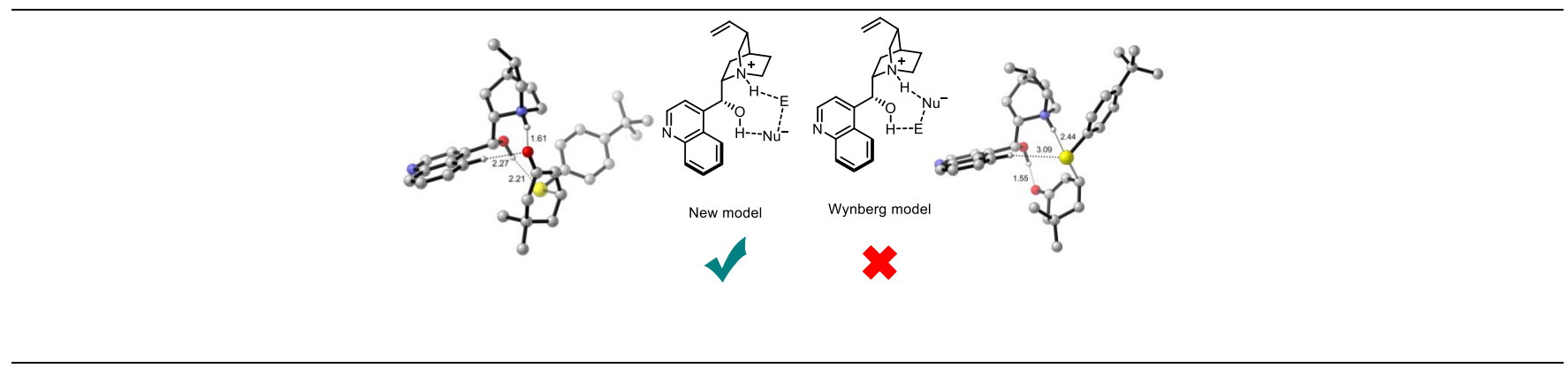

4 